\title{
Neutron-induced fission cross sections measurements at $\mathbf{n}_{-}$TOF
}

\author{
L. Audouin 1,2, a , L. Tassan-Got ${ }^{2,1}$, C. Stéphan ${ }^{2,1}$, C. Paradela ${ }^{2,3}$, B. Berthier ${ }^{2,1}$, L. Ferrant ${ }^{1,2}$, S. Isaev ${ }^{2}$, C. Le Naour ${ }^{2,1}$, \\ D. Trubert ${ }^{2,1}$, I. Duran ${ }^{3}$, and the $\mathrm{n}_{-}$TOF collaboration ${ }^{4}$ \\ 1 Université Paris XI, IPN Orsay, 91406 Orsay, France \\ 2 CNRS/IN2P3, UMR 8608, 91406 Orsay, France \\ 3 Universidad de Santiago de Compostela, Spain \\ ${ }^{4}$ www.cern.ch/ntof
}

\begin{abstract}
The neutron-induced fission cross sections of ${ }^{233} \mathrm{U},{ }^{234} \mathrm{U},{ }^{235} \mathrm{U},{ }^{238} \mathrm{U},{ }^{232} \mathrm{Th},{ }^{237} \mathrm{~Np},{ }^{209} \mathrm{Bi}$, ${ }^{\text {nat }} \mathrm{Pb}$ have been measured at the $n_{-}$TOF facility at CERN over 9 orders of magnitude in neutron energy using ${ }^{235} \mathrm{U}$ as a reference. Parallel Plate Avalanche Counters were used to detect both fission fragments in coincidence, thus efficiently discriminating fissions from other reactions. Data benefit from the remarkable energy resolution of the n_TOF facility. They are found in overall good agreement with databases and previous measurements, but some clear discrepancies can be put in evidence. These data are the first full coverage of the high-energy region (up to $1 \mathrm{GeV}$ ).
\end{abstract}

\section{Motivations}

Although the neutron-induced fission from some actinides like ${ }^{235} \mathrm{U}$ and ${ }^{238} \mathrm{U}$ has been extensively measured in the thermal and epithermal region up to a few $\mathrm{MeV}$ due to their key role in nuclear power, many nuclei that are important for the fuel cycle or for future reactors such as thorium-based reactors are not so well known. Furthermore, at higher energies, measurements are less numerous and beyond a few $\mathrm{MeV}$ they become scarce at best. The design of fast reactors requires precise knowledge of the nuclei behavior in the $\mathrm{MeV}$ region, and the design of Accelerator Driven System (ADS) extends such requirements to a few tens of $\mathrm{MeV}$ due to the very hard neutron spectra created by the ADS spallation source. In order to design such spallation sources, precise information about their constitutive material (lead and bismuth) are also required.

In a more fundamental point of view, it is well know that in the few tens of $\mathrm{MeV}$ energy region, fission reactions are not necessarily isotropic; but measurements of these phenomenons are very scarce and concern only a very few nuclei. Also, the transition from the compound nucleus regime toward the spallation regime has never been properly measured with neutrons, and the modeling of nuclear reactions could greatly benefit from the comparison of such neutron-induced reactions with the more well-known proton-induced reactions.

\section{Experimental facility}

Here we only recall the main features of the $n_{-}$TOF facility (see [1] for an extensive description). Bunches of $20 \mathrm{GeV}$ protons are shot every few seconds by the CERN Proton Synchrotron on a spallation target, thus producing neutrons over a wide range of energy (from the $\mathrm{MeV}$ region up to a few $\mathrm{GeV}$ ). A $5 \mathrm{~cm}$ water layer at the exit of the target acts as a moderator and strongly extends the neutron flux toward the thermal and epithermal region; figure 3 presents the resulting flux, as measured by our experimental system. The effective

\footnotetext{
${ }^{a}$ Presenting author, e-mail: audouin@ipno.in2p3.fr
}

neutron flux is in the order of a few $10^{5}$ neutrons per $\mathrm{cm}^{2}$ per proton bunch. The very low duty cycle of the facility leads to a very low neutron background. The combination of a $185 \mathrm{~m}$ flight path and the short duration of the proton pulse $(6 \mathrm{~ns})$ confer to the $\mathrm{n}_{-} \mathrm{TOF}$ facility an outstanding energy resolution, varying from $6 \%$ in the $\mathrm{GeV}$ region to $10^{-4}$ in the epithermal region.

In order to solve possible pile-ups at high energy and to fully benefit from the time resolution, all signals produced by detectors are digitised by 8 bits, $500 \mathrm{MHz}$ Flash Amplitude Digital Converters (FADC). The resulting data are stored for off-line analysis. Due to electronics constraints, data recording starts at the proton beam dump and ends after $16 \mathrm{~ms}$, a time that corresponds to the arrival of $0,7 \mathrm{eV}$ neutrons in the experimental area.

\section{Detection system and targets}

Proper fission measurements require to disentangle fissions from the $\alpha$ particles emitted by actinide targets and from the wide range of reaction channels (mostly spallation reactions in both the material of interest and its backing) that open beyond a few $\mathrm{MeV}$. An unambiguous tag from fission reactions consists in the simultaneous detection of both fission fragments. In order to achieve this, thin actinide targets were enclosed between PPACs as sketched on figure 1, each of the latter consisting of 3 thin foils and the resulting volumes being filled with 4 mbar $\mathrm{C}_{3} \mathrm{~F}_{8}$ gas. The experimental setup actually consisted in a stack of 10 PPACs interleaved with 9 targets. The neutron flux was perpendicular to detector foils and targets, and was not significantly altered as the neutron interaction probability never exceeded $10^{-4}$.

Each target consisted in a $8 \mathrm{~cm}$ diameter, $300 \mu \mathrm{g} . \mathrm{cm}^{-2}$ thick layer of the material of interest that was placed on a $600 \mu \mathrm{g} . \mathrm{cm}^{-2} \mathrm{Al}$ backing by electrodeposition. The $\alpha$ activity of some of these targets allowed a measurement of their homogeneity using $\alpha$ counting; for the less active materials, the RBS technique was used. The consistency between the 


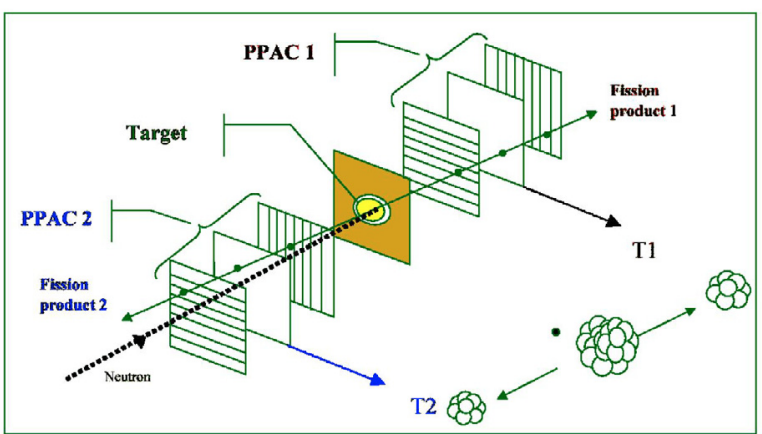

Fig. 1. Scheme of 2 PPACs enclosing a target. The stripped foils, combined with a delay-line readout, allow $\mathrm{X}$ and $\mathrm{Y}$ localisation in each detector, thus making possible a geometrical reconstruction of the fission event.

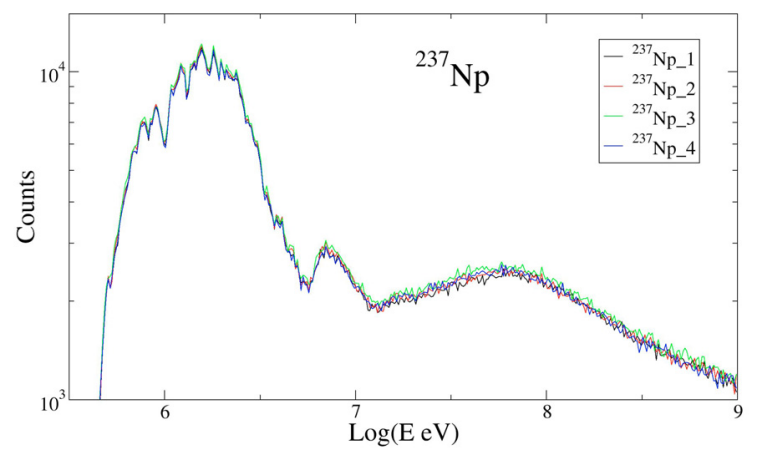

Fig. 2. Counting rate obtained using the anode signals only for 4 ${ }^{237} \mathrm{~Np}$ targets as a function of the logarithm of the neutron incident energy. Data are corrected for the different targets thickness.

two methods was successfully checked in the case of the ${ }^{235} \mathrm{U}$ target.

The localisation of each fission fragment is derived from the delays of the signals collected from the two ends of the delay line with respect to the fast anode signal. As the fragments emission is (at least at low energy) a back to back phenomena, the knowledge of the two fragments positions allows a full determination of the emitting point on the target and the fission angle. This angle plays a significant role in the detection efficiency as the energy stopping in the target and detector materials induces a limiting detection angle. This energy stopping also depends on mass splitting, which changes with the incident neutron energy (the actinide fission is asymmetric at low energy and becomes slowly symmetric as the energy reaches the tens of $\mathrm{MeV}$ region).

A systematic measurement of the localisation results in a complete control of the detectors efficiency and makes possible a measurement of the targets inhomogeneities, of the beam position on the targets, as well as a measurement of fission anisotropies. Due to electronic problems, such a localisation measurement was not performed with the required efficiency and therefore could not be used for cross sections measurements, which rely only on the coincidences of the fast anode signals. However, as it can be seen in the figure 2, the counting rates obtained with this method for 4 different ${ }^{237} \mathrm{~Np}$ targets are in good agreement; the inconsistencies between these rates indeed provide an excellent estimation of the systematic error on the counting rates. We evaluate this error to $5 \%$ below the $\mathrm{MeV}$ region, $10 \%$ in the tens of $\mathrm{MeV}$ region (this is a conservative value aimed at accounting for the possible different variations of the fission anisotropy from one nuclei to the other), and $7 \%$ be yond $100 \mathrm{MeV}$ (again a conservative value taking into account the efficiency variations caused by the recoil imparted to the fissioning nucleus by the momentum transfer).

\section{Normalisation of counting rates}

At the moment, no absolute measurement of the neutron flux is available at $n_{-}$TOF. Therefore the determination of all cross sections requires a normalisation to a reference target. For fission measurements, a ${ }^{235} \mathrm{U}$ target was considered as a reference target for normalisation purposes and was always present during measurements. Fission cross sections $\sigma$ for nuclei $X$ can then be deduced at each neutron energy from the counting rates $C$ using the trivial formula:

$$
\sigma_{n_{-} T O F}(X)=\frac{C(X)}{C\left({ }^{235} \mathrm{U}\right)} \sigma_{\text {reference }}\left({ }^{235} \mathrm{U}\right) .
$$

The reference ${ }^{235} \mathrm{U}$ cross section on the whole $1 \mathrm{eV}-1 \mathrm{GeV}$ range was constructed in three parts:

- up to $20 \mathrm{MeV}$, ENDF-B6;

- 20 to $200 \mathrm{MeV}$, the High Energy extension of JENDL [2];

- beyond $200 \mathrm{MeV}$, the value at $200 \mathrm{MeV}(1420 \mathrm{mb})$.

Using this reference cross section and the measured counting rate for the ${ }^{235} \mathrm{U}$ target, it is possible to extract an estimation of the neutron flux. This flux is presented on figure 3. In the energy range for which the ${ }^{6} \mathrm{Li}$ neutron detector is qualified (below $1 \mathrm{MeV}$ ), the agreement between the shapes obtained by the 2 methods is excellent.

\section{Results}

First of all, it is worth pointing out that we have measured fission cross sections on 9 orders of magnitude in neutron

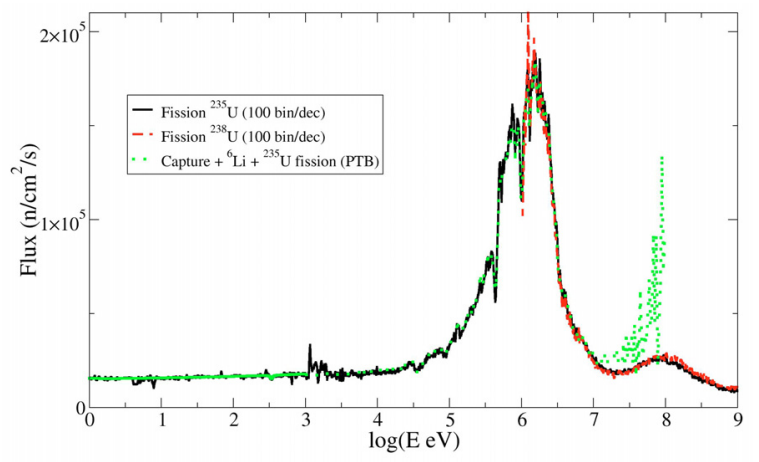

Fig. 3. The $n_{-}$TOF flux distribution as deduced from ${ }^{235} \mathrm{U}$ fission, ${ }^{238} \mathrm{U}$ fission, and a ${ }^{6} \mathrm{Li}$ monitor coupled to a fission chamber. Note the fluctuations in the ${ }^{235} \mathrm{U}$ around $10 \mathrm{keV}$ : they are the consequence of resonances that are missing in the database. 
energy in a single measurement. Figure 4 displays the results for ${ }^{237} \mathrm{~Np}$. One can clearly see the subthreshold resonance region that extends up to $1 \mathrm{MeV}$, the first and second chance fission regions that extend up to $50 \mathrm{MeV}$, and the spallation region beyond this.

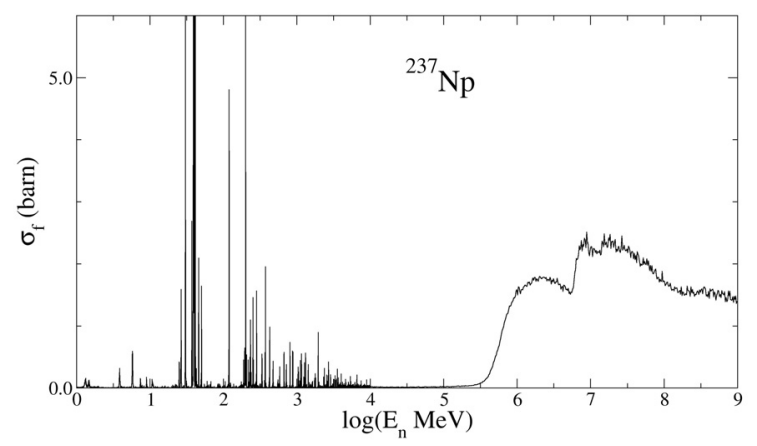

Fig. 4. Neutron-induced fission cross section on ${ }^{237} \mathrm{~Np}$ measured at $\mathrm{n}_{-} \mathrm{TOF}$ as a function of the $\log$ of the neutron energy.
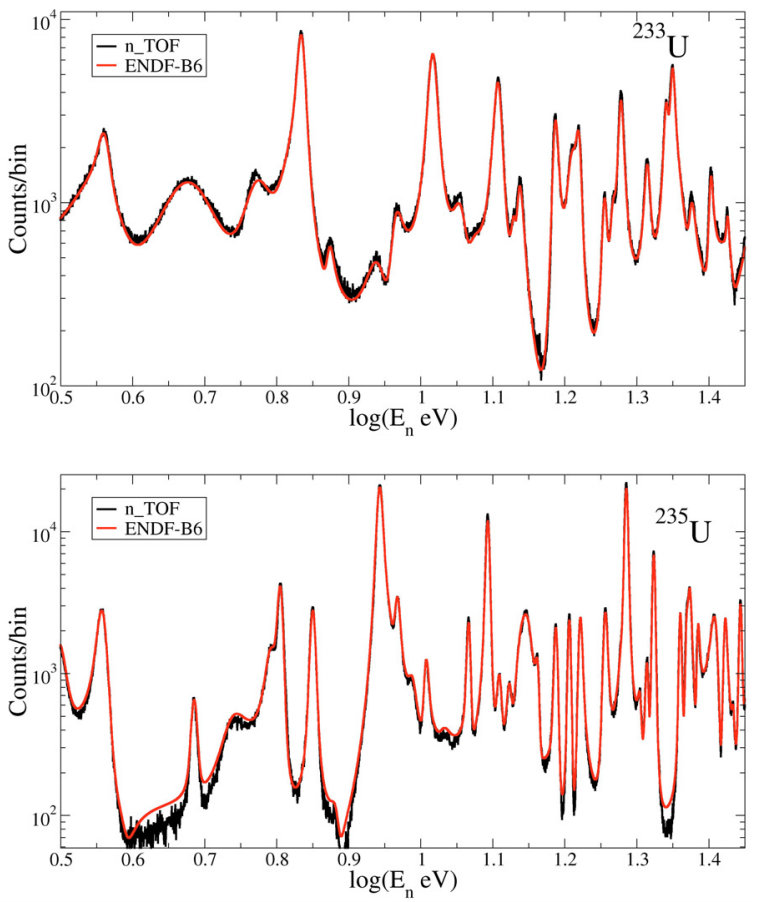

Fig. 5. Upper figure: fission counting rate measured at $\mathrm{n}_{-} \mathrm{TOF}$ on ${ }^{233} \mathrm{U}$ (continuous line) as a function of the neutron energy, compared to ENDF-B6 values (dashed line) - see text for details. Lower figure: same for ${ }^{235} \mathrm{U}$.

We have already indicated that due to its very low duty cycle, the n_TOF facility has a very low neutron background. This can be put in evidence in the low energy region, where neutron background is expected to have its largest contribution, by comparing the count rate on a well-known nuclei like ${ }^{233} \mathrm{U}$ with an evaluated database renormalised using a constant factor (the flux being flat in this short energy range). This
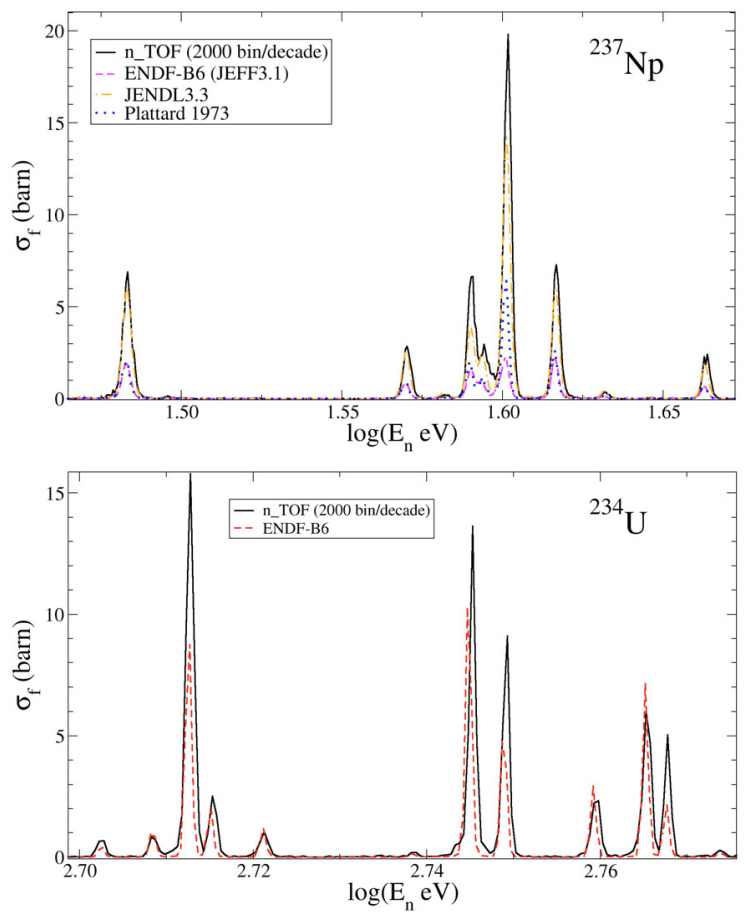

Fig. 6. Lower figure: fission cross section measured at $n_{-}$TOF on ${ }^{234} U$ (continuous line) compared to ENDF-B6 values (dashed line) as a function of the logarithm of the neutron energy. Upper figure: same for ${ }^{237} \mathrm{~Np}$, with additional plotting of JENDL-3.3 (dashed-dotted line) and Plattar measurement (dotted line).

comparison is presented in figure 5 (upper part): the agreement is excellent, even in the dips, which indeed illustrates the very low neutron background. The same comparison can be made for the ${ }^{235} U$ target (lower part of figure 5). In this case, a fine agreement is obtained for all resonances, but in the deeps, our data are lower by up to $30 \%$. These preliminary results indicate that the $\mathrm{n}_{-} \mathrm{TOF}$ measurement has been carried out with better neutron background conditions and statistics than previous measurements.

In the case of ${ }^{237} \mathrm{~Np}$ and ${ }^{234} \mathrm{U}$, there are clear discrepancies between our data and evaluated databases in the subthreshold resonances region (see figure 6). Some of these resonances from ${ }^{237} \mathrm{~Np}$ appear to be underestimated by factors between 5 and 10 in ENDF-B6, and by $25 \%$ in JENDL-3.3 (upper figure). Discrepancies are more reduced, while still significant, in the case of ${ }^{234} \mathrm{U}$ resonances.

At higher energy, the ratio of fission cross sections of ${ }^{238} \mathrm{U}$ and ${ }^{235} \mathrm{U}$ is an interesting test case, as in our case, this quantity is independent of the neutron flux because the measurements on both nuclei were performed simultaneously. Our results are presented in figure 7 . We find out that our data are very close to the ENDF-B6 + JENDL-HE base (note for example the nice agreement in the delicate shape associated with the 2 nd chance opening), with a systematical shift of about $+5 \%$. Although this shift is smaller than our estimated systematic uncertainty, it is worth noticing that the preliminary results obtained by FIC [3], also at n_TOF, show the same tendency. If we compare our results to previous measurements, we find the same slight shift with the work of Lisowski (on which the evaluations largely 


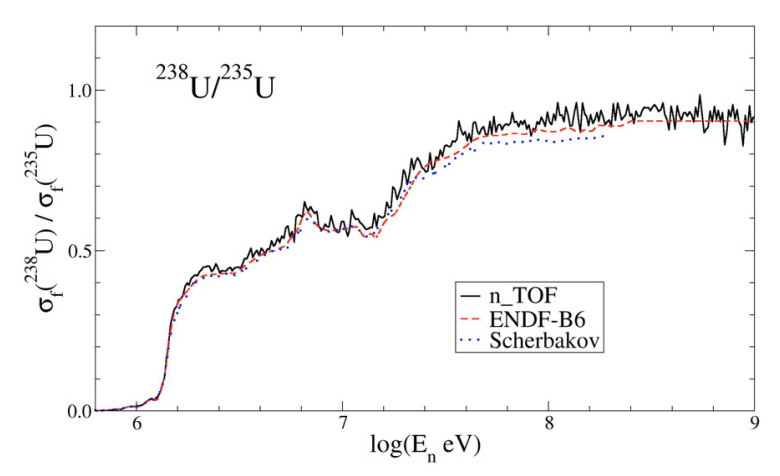

Fig. 7. Ratio of the fission cross sections measured at $\mathrm{n}_{-} \mathrm{TOF}$ for the ${ }^{238} \mathrm{U}$ and the ${ }^{235} \mathrm{U}$ targets, as a function of the $\log$ of the neutron energy, compared to ENDF-B6 (dashed line) and Shcherbakov measurements (dotted line).
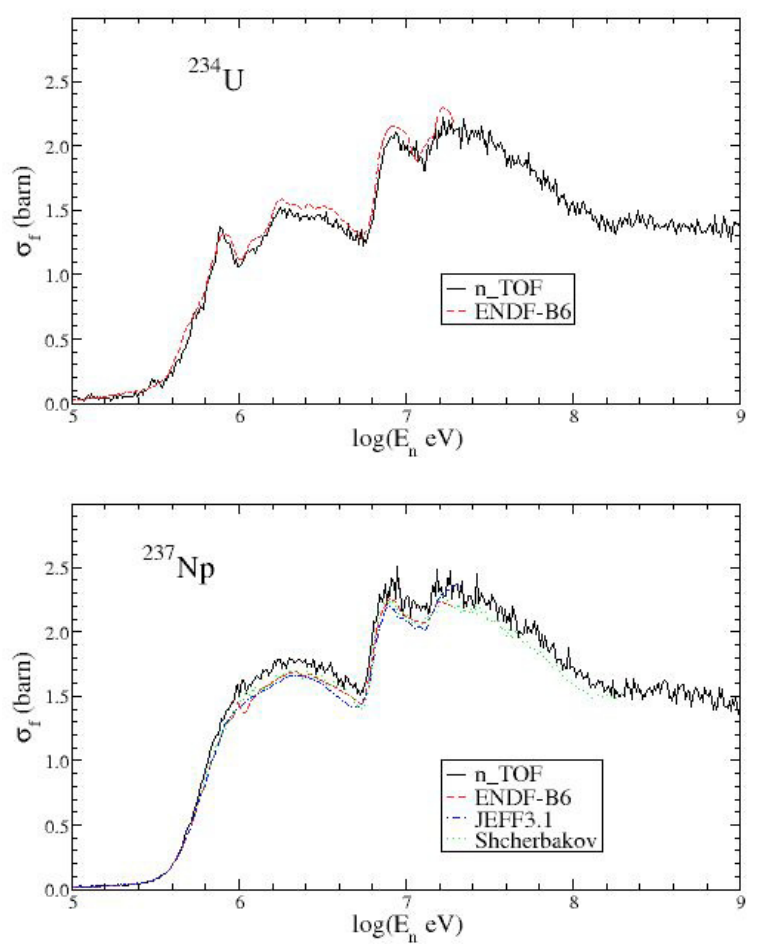

Fig. 8. Upper figure: fission cross section measured at $n_{-}$TOF on ${ }^{234} \mathrm{U}$ (continuous line) compared to ENDF-B6 values (dashed line) as a function of the logarithm of the neutron energy. Lower figure: same measurement on ${ }^{237} \mathrm{~Np}$ (continuous line) this time compared to ENDF-B6 values (dashed line), JEFF-3.1 (dashed-dotted line) and Shcherbakov measurement (dotted line) (the 3 later are highly similar and hence hardly distinguishable here).

rely), and Shcherbakov [4] beyond $20 \mathrm{MeV}$. It is difficult to draw solid conclusions from such discrepancies as all these results are compatible within their respective error bars.
For two other threshold nuclei $\left({ }^{234} \mathrm{U}\right.$ and $\left.{ }^{237} \mathrm{~Np}\right)$, our data exhibit trends very similar to evaluated databases or previous measurements (see figure 8); they are compatible within a 7\% error. As in the case of the U8/U5 ratio, a small systematic shift is observed, $+5 \%$ in the case of ${ }^{237} \mathrm{~Np},-5 \%$ in the case of ${ }^{234} \mathrm{U}$. These different trends may indicate that the ${ }^{235} \mathrm{U}$ normalisation is correct. Again, let us point out that all our targets were measured simultaneously, and that the efficiency of our detectors is found to be very close from one detector to the other (see section 3), so it seems unlikely that we systematically underestimated or overestimated the fission cross section of one sample or another.

\section{Summary and perspectives}

The neutron-induced fission cross section of ${ }^{232} \mathrm{Th},{ }^{233} \mathrm{U},{ }^{234} \mathrm{U}$, ${ }^{235} \mathrm{U},{ }^{238} \mathrm{U},{ }^{237} \mathrm{~Np}$, ${ }^{\text {nat }} \mathrm{Pb},{ }^{209} \mathrm{Bi}$ have been measured at the $\mathrm{n}_{-}$TOF facility (CERN) relatively to a ${ }^{235} \mathrm{U}$ target by detecting both fission fragments in coincidence. This method has been shown as being fully effective in order to discard $\alpha$ emissions and the products of high-energy reactions. Our results extend over 9 orders of magnitude in neutron energy (from $1 \mathrm{eV}$ to $1 \mathrm{GeV}$ ) and we estimate the systematic error on the cross sections to vary between 7 and $12 \%$ depending on the energy range.

Overall, an excellent agreement is obtained with available databases, especially in the thermal and epithermal regions where a large amount of experimental data exist. However, some interesting discrepancies can be noticed, such as the amplitude of subthreshold resonances in ${ }^{237} \mathrm{~Np}$. Beyond $10 \mathrm{MeV}$, systematic shifts of about $\pm 5 \%$ are also noticed in comparison to evaluated databases; however the cross-check between targets containing the same isotope is a strong argument in favor of the self-consistency of our results.

Future measurements will focus on more radioactive targets such as Am isotopes. We also plan to dedicate measurements to the fission anisotropy phenomenon, which will be of interest for the theoretical understanding of the fission and will also help to reduce the uncertainties on our data in the 10 to $100 \mathrm{MeV}$ region. Absolute fission measurements would be highly relevant, especially for the high energy part; but such a measurement requires an appropriate neutron detector, probably based on the (n,p) diffusion for which the cross sections are very well known. However such a detector remains to be conceived for the $n_{-}$TOF facility.

\section{References}

1. C. Borcea et al., NIM A 513, 524 (2003).

2. A.Y. Konobeyev et al., JAERI Research Report 2002-28.

3. M. Calviani (these proceedings).

4. O.A. Shcherbakov et al., ISINN-9, Dubna (2001). 